\title{
Sinonasal Renal Cell-Like Carcinoma: Case Report and Review of the Literature
}

\author{
Mark Kubik $^{1}\left(\mathbb{D} \cdot\right.$ Nicholas Barasch $^{2} \cdot$ Garret Choby $^{1} \cdot$ Raja Seethala $^{2}$. \\ Carl Snyderman ${ }^{1}$
}

Received: 9 October 2016 / Accepted: 16 December 2016 / Published online: 28 December 2016

(C) Springer Science+Business Media New York 2016

\begin{abstract}
The differential diagnosis for clear cell neoplasms of the sinonasal tract is wide but critical to understand. In this paper, we describe a case of renal cell-like adenocarcinoma (SNRCLA) presenting as a paranasal sinus mass with clear cell morphology. The relevant literature is reviewed and the workup for clear cell neoplasms described. SNRCLA is a rarely encountered low grade glandular malignancy of the paranasal sinuses. Despite its morphologic mimicry of renal cell carcinoma, SNRCLA demonstrates a seromucinous phenotype and is associated with a favorable prognosis and low recurrence rates after surgical resection.
\end{abstract}

\section{Introduction}

Malignant tumors of the sinonasal tract are relatively rare clinical entities. Primary sinonasal malignancies generally comprise less than $3 \%$ of all head and neck malignancies and the incidence has been estimated to be on the order of 0.5-1.0 per 100,000 people $[1,2]$. In addition to their rarity, these tumors are extraordinarily diverse with respect to their pathologic characteristics, risk factors, clinical behavior, and response to surgical or systemic therapy. The most

M. Kubik and N. Barasch contributed equally to this work and share first authorship status.

Mark Kubik

kubikmw@upmc.edu

1 Department of Otolaryngology, Center for Cranial Base Surgery, University of Pittsburgh Medical Center, 200 Lothrop Street, EEI 521, Pittsburgh, PA 15213, USA

2 Department of Pathology, University of Pittsburgh Medical Center, Pittsburgh, PA, USA recent WHO classification of primary sinonasal malignancies contains 44 distinct histopathologic entities [3]. Understandably, these cancers present a formidable challenge for both the surgeon and pathologist.

In this article we present an unusual case of clear cell neoplasia of the paranasal sinuses found to be consistent with sinonasal renal cell-like adenocarcinoma (SNRCLA). The finding of clear cell neoplasia in a sinonasal specimen is rare but associated with a surprisingly wide differential diagnosis. When evaluating the differential diagnosis for a clear cell neoplasm of the sinonasal tract, an underlying renal cell carcinoma (RCC) must first be ruled out. RCC is the most common metastatic lesion to the nasal cavity and paranasal sinuses [4] and approximately $6-15 \%$ of patients with RCC will have metastatic deposits in the head and neck [5, 6]. In addition to metastatic RCC, other diagnostic considerations for clear cell neoplasia in the paranasal sinuses include a clear cell variant of squamous cell carcinoma, melanoma, primary salivary clear cell carcinoma, metastatic clear cell thyroid carcinoma, clear cell predominant mucoepidermoid carcinoma, and SNRCLA.

\section{Case Report}

An 80 year old female presented to our otolaryngology clinic with an asymptomatic right sinonasal mass. She reported a history of left sided acinic cell carcinoma treated at an outside institution with endonasal resection and adjuvant radiotherapy approximately eight years prior. Diagnostic nasal endoscopy revealed a large friable soft tissue mass arising from the lateral nasal wall near the axilla of the right middle turbinate. Imaging revealed a $3 \mathrm{~cm}$ tumor centered at the right nasolacrimal sac with extension superiorly to involve the nasal septum and dura of the anterior 


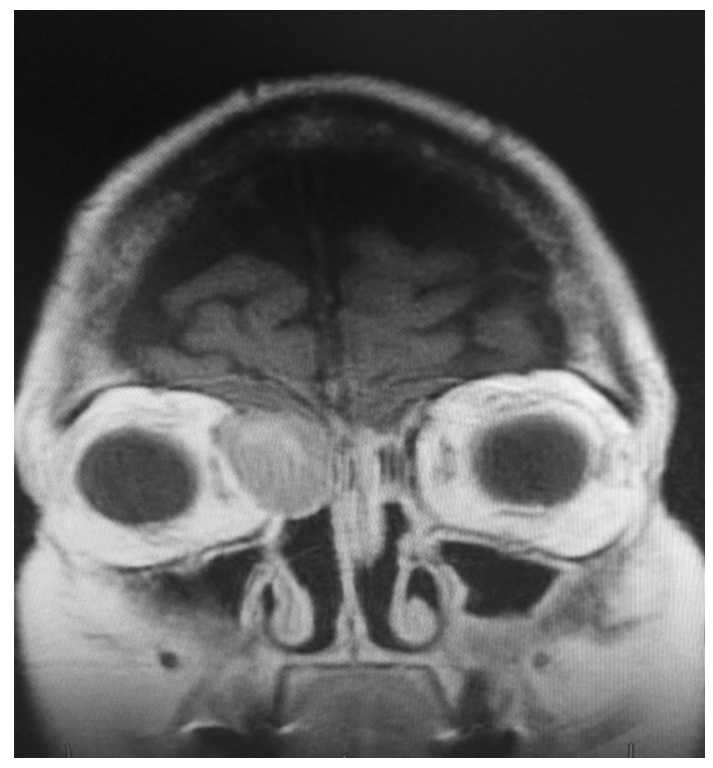

Fig. 1 Coronal T1 weighted MRI with gadolinium showing an enhancing mass centered in the right medial orbit and anterior skull base

skull base (See Fig. 1). CT imaging of the neck and chest revealed no evidence of cervical lymphadenopathy or metastatic disease. Given the location and extent of the tumor, a combined endoscopic and open craniofacial approach was pursued. The patient underwent a right transfrontal orbital approach with craniectomy performed through a bicoronal incision. This included removal of both involved dura and medial periorbita as margins. Reconstruction involved a pericardial dural graft and pericranial flap. The patient did well postoperatively without any complications. Pathologic examination demonstrated a clear cell neoplasm with hemorrhage and prominent vasculature (Fig. 2a). Tumor cells were polygonal with distinct cell membranes (Fig. 2b). While most cells demonstrated clear cytoplasm, some were smaller with slightly eosinophilic cytoplasm. Mild to moderate nuclear size variation is noted, without prominent nucleoli. Overall the morphologic appearance was reminiscent of a metastatic renal cell carcinoma. However, the tumor cells were positive for SOX-10, DOG1 (weak membranous fashion), CK7, and negative for PAX-8. PAS after diastase showed very focal granular staining, though not prominent enough to confirm a "zymogen granule" pattern of staining (Fig. 2c-f). Based on the morphology and immunophenotype, a diagnosis of sinonasal renal cell like carcinoma was rendered. All resection margins were negative for tumor. Given the low grade histology, margin negative resection, and risks of re-irradiation, the decision was made against adjuvant therapy. Also, given the unique nature of the pathology, the patient underwent a CT scan of the abdomen to exclude a primary renal neoplasm which was normal.

\section{Discussion}

SNRCLA is a relatively new entity that was first described in in 2002 when Zur et al. described a patient with a sinonasal clear cell carcinoma resembling renal cell carcinoma in the absence of any underlying primary renal tumor [7]. Since that initial description, additional reports have surfaced and currently there are 13 patients described in the
Fig. 2 Sinonasal Renal CellLike Carcinoma (Histopathologic Features) Characteristic nested growth of polygonal clear cells with intervening fibrovascular stroma and hemorrhage (a). No necrosis, angiolymphatic invasion, perineural invasion, or mitoses are present. Interspersed cells with slightly eosinophilic cytoplasm, low nuclear:cytoplasmic ratio, and minimal size variation are present (b). PASd does not highlight zymogen granules (b insert). Variable faint DOG1 membranous staining (c). Diffuse nuclear SOX10 staining (d). Strong membranous and cytoplasmic CK7 staining (e). Negative Pax- 8 staining (f). The tumor is also negative for TTF1, CD10, CK20, mucicarmine, and mammaglobin

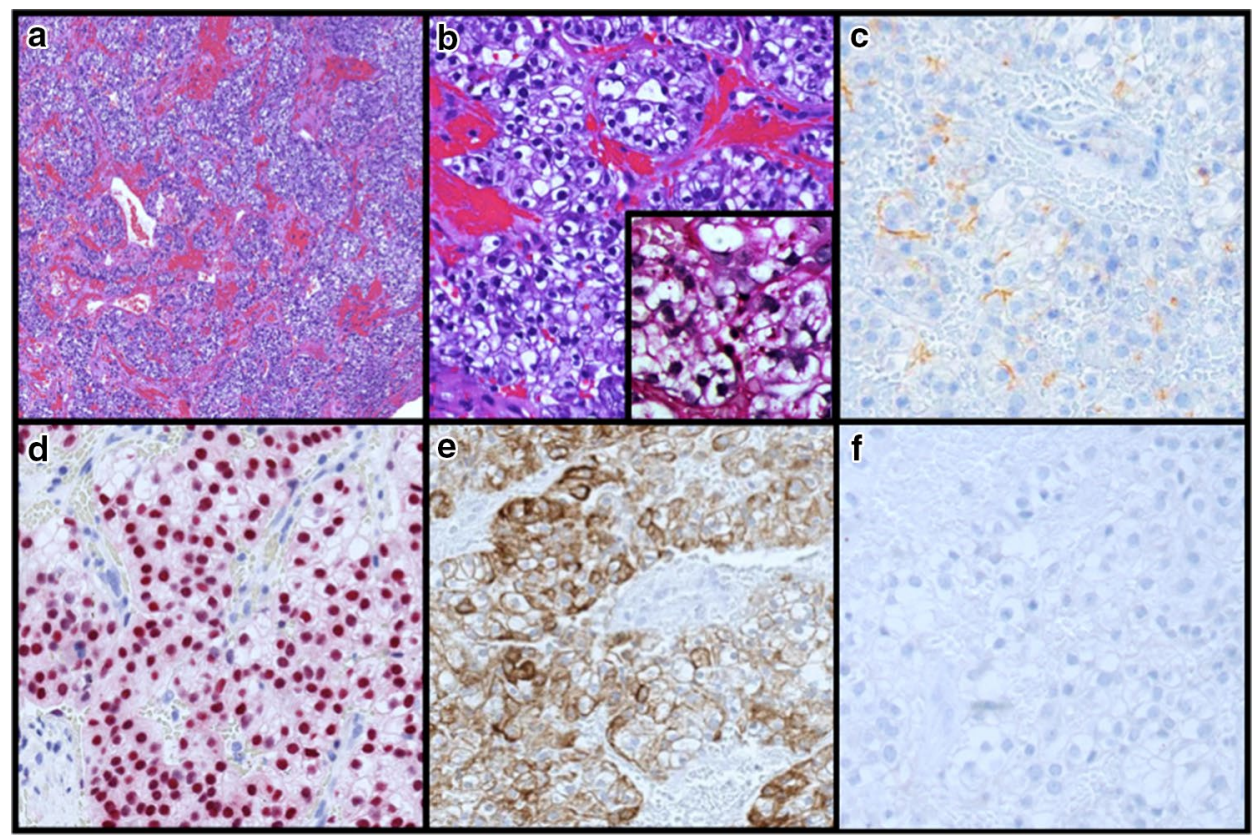


literature with similar clinical and histopathologic findings [8].

A review of the literature demonstrates a female predilection of 9:4 and an age range of 22 to 77 years. Symptoms on presentation are similar to other sinonasal tumors and have included epistaxis and nasal obstruction. Endoscopically, this tumor has been described in most articles to be a red, polypoid appearing mass. Tumor subsites have included the following: nasal cavity $(\mathrm{N}=9)$, paranasal sinuses $(\mathrm{N}=2)$, nasopharynx $(\mathrm{N}=2)$ [7-10]. This case is unique in that it occurred 8 years after radiation therapy for a contralateral "acinic cell carcinoma." The original materials were not available for our review, thus any relationship between this tumor and the current one is speculative. Interestingly however, the contralateral location raises the possibility of a second primary rather than a recurrence despite the potential for morphologic overlap between an acinic cell carcinoma and SNRLCA.

Histologic features of SNRLCA include a low grade clear cell neoplasm with large polygonal cells, intratumoral hemorrhage, and low nuclear:cytoplasmic ratio resembling primary renal cell carcinoma. The tumor can be organized in solid, nodular, or follicular forms with rare mitotic activity. Extension invasion and necrosis are not typically seen $[7,8,11,12]$.

Phenotypically, SNRLCA expresses epithelial markers such as CK7 and frequently will not express CK20 [9]. Both vimentin, which stains the intermediate filaments of tissues of mesenchymal origin, and Pax-8, a marker of mullerian, renal and thyroid tissue, are not expressed in SNRLCA [13]. While the cell of origin of SNRLCA is not established, $[7,8,10,11]$ DOG-1 and SOX-10 expression confirm the seromucinous phenotype of SNRLCA. Even based on the earlier cases, a seromucinous phenotype was implied given the frequent S100 immunoreactivity, and carbonic anhydrase expression typical of Scheiderian seromucinous glands [10]. One previous case tested for DOG-1 [10] was reportedly negative, but here it is positive along with SOX-10. DOG-1, a calcium dependent chloride channel, demonstrates moderate apical staining in the sinonasal seromucinous glands $[14,15]$. Similarly SOX-10 is strongly expressed insinonasal glands [14]. Thus SNRLCA is likely simply a clear cell variant of a low grade seromucinous type sinonasal non intestinal type adenocarcinoma.

There are several tumors that must be considered in the differential diagnosis of a tumor of the sinonasal region with clear cell morphology. While some can be ruled out by morphologic features, immunohistochemical studies can be beneficial in sorting out the morphologically similar lesions. The approach must address the possibility of primary salivary clear cell, primary non-salivary clear cell leions, and metastatic clear cell lesions.

\section{Primary (Named) Salivary Clear Cell Lesions}

The aforementioned seromucinous phenotype raises consideration for named salivary clear cell tumors [7, 8]. A major diagnostic challenge given the SOX-10 and DOG-1 immunoreactivity will be in distinguishing the lesion from acinic cell carcinoma. Acinic cell carcinoma can occur in the sinonasal tract and may occasionally show clear cell morphology and, in our case, there is rare granular staining with PASD, but not to the degree that would confirm true zymogen granules. Additionally, clear cell morphology is generally only focally noted in acinic cell carcinomas in contrast to its predominance here in this SNRCLA [7-9]. Finally, DOG-1 is generally far more intense in acinic cell carcinoma than SNRCLA [14]. Clear cell mucoepidermoid carcinoma may show some overlap, but consists of mucous, intermediate and epidermoid cell types, the latter two of which are more diffusely p63 positive [10]. Hyalinizing clear cell carcinoma may also be considered; however it is rare in the sinonasal tract, has a distinctive fibrocellular stroma juxtaposed to peritumoral hyaline material, and is phenotypically squamous and thus p63 positive $[10,11]$. Also, while not a necessary test in this case, hyalinizing clear cell carcinomas are further defined by EWSRI-ATF1 translocation, and thus SNRCLA are expected to be negative for this. Other clear cell neoplasms to consider include clear cell oncocytoma, clear cell myoepithelial carcinoma, and epithelial myoepithelial carcinoma. The former does not show SOX-10 or DOG-1 reactivity, while the latter two will demonstrate other myoepithelial marker expression.

\section{Primary Non-Salivary Clear Cell Lesions}

Squamous cell carcinoma (SCC) with clear cell differentiation can occur as a primary lesion in the head and neck. However, as in mucoepidermoid carcinoma, the lack of squamous features or strong p63 reactivity makes this diagnosis unlikely. Furthermore, the relative weakness of p63 staining strongly discourages a diagnosis of SCC [10]. Endolymphatic sac tumors could also be considered based on morphology; however, they occur in the inner ear/skull base region and have papillary fronds which are not present in this tumor [10]. Paragangliomas can have clear cell morphology and can also rarely manifest in the sinonasal region. The absence of the more nested architecture, "salt and pepper" chromatin, as well as a diffuse rather than sustentacular SOX-10 staining profile and cytokeratin positivity argue against this diagnosis. While not performed here, further workup demonstrating negativity with synaptophysin and chromogranin would definitively rule out a paraganglioma [7]. 


\section{Metastatic Clear Cell Lesions}

Based on the clear cell appearance, nuclear morphology, and hemorrhage, the most important metastatic lesion that should be considered is clear cell RCC. The namesake of the SNRCLA, clear cell RCC is documented to metastasize to the head and neck [6]. Clear cell RCC are characteristically CD10, vimentin, and PAX-8 positive. RCC marker is also useful when positive, but is less sensitive. In this case, the CK7 and SOX-10 positivity and PAX-8 negativity, as was the case with our lesion argue against a metastatic clear cell RCC [7, 16, 17]. Metastatic melanoma, specifically with balloon cell morphology, and clear cell sarcoma (formerly clear cell melanoma of soft parts), are less likely given the strong CK7 positivity. The clear cell thyroid carcinomas are a consideration but would be expected to demonstrate TTF- 1 and PAX-8 positivity [10]. PEComas are rare in the sinonasal tract and are typically keratin negative. Also, though they often express various myoid and melanocytic markers, they are usually S100 and SOX-10 negative [18, 19].

Limited published clinical experience with this tumor suggests it is a low-grade carcinoma with an indolent clinical course. Of the thirteen patients described in the literature, only one patient has been described with locally recurrent disease [20]. No patients in any of these series had metastatic disease to the neck or distant sites. Management strategies described have generally included endoscopic or open surgical resection followed by adjuvant radiotherapy. However, three patients have been treated with primary chemoradiation therapy successfully without evidence of recurrent disease. The indolent course of SNRCLA is in stark contrast to other clear cell malignancies of the nasal cavity including melanoma, clear cell salivary tumors, and metastatic renal cell carcinoma, all of which have a decidedly more aggressive natural history.

\section{Conclusion}

SNRCLA is a rare but distinct glandular malignancy of the sinonasal tract. It should be included in the differential diagnosis when a sinonasal clear cell neoplasm is identified. In contrast to other clear cell neoplasms of the sinonasal tract, early reports of the clinical behavior and natural history suggest that this is a low-grade neoplasm with a favorable response to surgical and nonsurgical treatment modalities.

Funding This study was not funded.

\section{Compliance with Ethical Standards}

Conflicts of interest All authors declare that they have no conflicts of interest to report.

Ethical Approval The patient described in this case study provided verbal and written informed consent for the publication of this manuscript.

\section{References}

1. Muir CS, Nectoux J. Descriptive epidemiology of malignant neoplasms of nose, nasal cavities, middle ear and accessory sinuses. Clin Otolaryngol Allied Sci. 1980;5(3):195-211.

2. Dulguerov P, Jacobsen MS, Allal AS, Lehmann W, Calcaterra T. Nasal and paranasal sinus carcinoma: are we making progress? A series of 220 patients and a systematic review. Cancer. 2001;92(12):3012-29.

3. Barnes L, Tse L, Hunt J. World Health Organization Classification of Tumors. Pathology and Genetics, Head and Neck Tumors. Lyon: IARC Press; 2005.

4. Bernstein JM, Montgomery WW, Balogh KJ. Metastatic tumors to the maxilla, nose, and paranasal sinuses. Laryngoscope. 1966;76(4):621-50.

5. Flocks RH, Boatman DL. Incidence of head and neck metastases from genito-urinary neoplasms. Laryngoscope. 1973;83(9):1527-39.

6. Remenschneider AK, Sadow PM, Lin DT, Gray ST. Metastatic renal cell carcinoma to the sinonasal cavity: a case series. J Neurol Surg Rep. 2013;74(2):67-72.

7. Zur KB, Brandwein M, Wang B, Som P, Gordon R, Urken ML. Primary description of a new entity, renal cell-like carcinoma of the nasal cavity: van Meegeren in the house of Vermeer. Arch Otolaryngol Head Neck Surg. 2002;128(4):441-7.

8. Bishop JA. Newly Described Tumor Entities in Sinonasal Tract Pathology. Head Neck Pathol. 2016;10(1):23-31.

9. Baghirath PV, Kumar JV, Vinay BH. Hyalinizing clear cell carcinoma: A rare entity. J Oral Maxillofac Pathol JOMFP. 2011;15(3):335-9.

10. Shen T, Shi Q, Velosa C, et al. Sinonasal renal cell-like adenocarcinomas: robust carbonic anhydrase expression. Hum Pathol. 2015;46(11):1598-606.

11. Storck K, Hadi UM, Simpson R, Ramer M, Brandwein-Gensler M. Sinonasal renal cell-like adenocarcinoma: a report on four patients. Head Neck Pathol. 2008;2(2):75-80.

12. Brandwein-Gensler M, Wei S. Envisioning the next WHO head and neck classification. Head Neck Pathol. 2014;8(1):1.

13. Butler RT, Alderman MA, Thompson LDR, McHugh JB. Evaluation of PAX2 and PAX8 expression in salivary gland neoplasms. Head Neck Pathol. 2015;9(1):47-50.

14. Chenevert J, Duvvuri U, Chiosea S, et al. DOG1: a novel marker of salivary acinar and intercalated duct differentiation. Mod Pathol Off J U S Can Acad Pathol Inc. 2012;25(7):919-29.

15. Purgina B, Bastaki JM, Duvvuri U, Seethala RR. A Subset of Sinonasal Non-Intestinal Type Adenocarcinomas are Truly Seromucinous Adenocarcinomas: A Morphologic and Immunophenotypic Assessment and Description of a Novel Pitfall. Head Neck Pathol. 2015;9(4):436.

16. Weinreb I. Hyalinizing clear cell carcinoma of salivary gland: a review and update. Head Neck Pathol. 2013;7(Suppl 1):S20.

17. Leivo I. Sinonasal Adenocarcinoma: Update on Classification, Immunophenotype and Molecular Features. Head Neck Pathol. 2016;10(1):68-74. 
18. Mai KT, Belanger EC. Perivascular epithelioid cell tumour (PEComa) of the soft tissue. Pathology (Phila). 2006;38(5):415.

19. Miettinen M, McCue PA, Sarlomo-Rikala M, et al. Sox10-a marker for not only schwannian and melanocytic neoplasms but also myoepithelial cell tumors of soft tissue: a systematic analysis of 5134 tumors. Am J Surg Pathol. 2015;39(6):826.
20. Heffner DK, Hyams VJ, Hauck KW, Lingeman C. Low-grade adenocarcinoma of the nasal cavity and paranasal sinuses. Cancer. 1982;50(2):312-2. 